\title{
(- OPEN ACCESS \\ Patient complaints in healthcare systems: a systematic review and coding taxonomy
}

\author{
Tom W Reader, Alex Gillespie, Jane Roberts
}

Department of Social Psychology, London School of Economics, London, UK

\section{Correspondence to} Dr Tom Reader, Department of Social Psychology, London School of Economics, Houghton Street, London WC2A 2AE, UK; t.w.reader@|se.ac.uk

Received 27 August 2013 Revised 14 March 2014 Accepted 4 May 2014 Published Online First 29 May 2014

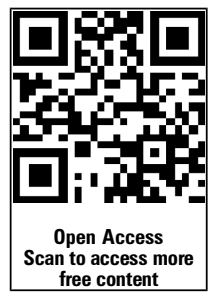

CrossMark

To cite: Reader TW,

Gillespie A, Roberts J. BMJ

Qual Saf 2014;23:678-689.

\begin{abstract}
Background Patient complaints have been identified as a valuable resource for monitoring and improving patient safety. This article critically reviews the literature on patient complaints, and synthesises the research findings to develop a coding taxonomy for analysing patient complaints. Methods The PubMed, Science Direct and Medline databases were systematically investigated to identify patient complaint research studies. Publications were included if they reported primary quantitative data on the content of patientinitiated complaints. Data were extracted and synthesised on (1) basic study characteristics; (2) methodological details; and (3) the issues patients complained about.
\end{abstract}

Results 59 studies, reporting 88069 patient complaints, were included. Patient complaint coding methodologies varied considerably (eg, in attributing single or multiple causes to complaints). In total, 113551 issues were found to underlie the patient complaints. These were analysed using 205 different analytical codes which when combined represented 29 subcategories of complaint issue. The most common issues complained about were 'treatment' (15.6\%) and 'communication' (13.7\%). To develop a patient complaint coding taxonomy, the subcategories were thematically grouped into seven categories, and then three conceptually distinct domains. The first domain related to complaints on the safety and quality of clinical care (representing 33.7\% of complaint issues), the second to the management of healthcare organisations (35.1\%) and the third to problems in healthcare staff-patient relationships (29.1\%).

Conclusions Rigorous analyses of patient complaints will help to identify problems in patient safety. To achieve this, it is necessary to standardise how patient complaints are analysed and interpreted. Through synthesising data from 59 patient complaint studies, we propose a coding taxonomy for supporting future research and practice in the analysis of patient complaint data.

\section{INTRODUCTION}

Patient complaints provide a valuable source of insight into safety-related problems within healthcare organisations. ${ }^{1}$ Patients are sensitive to, and able to recognise, a range of problems in healthcare delivery, ${ }^{2}$ some of which are not identified by traditional systems of healthcare monitoring (eg, incident reporting systems, retrospective case reviews). ${ }^{3}$ Thus, patient complaints can provide important and additional information to healthcare organisations on how to improve patient safety. ${ }^{4}$ Furthermore, analysing data on negative patient experiences strengthens the ability of healthcare organisations to detect systematic problems in care. This has recently been highlighted in the UK through the Francis report ${ }^{5}$ on 1200 unnecessary deaths that occurred over 3 years at Mid-Staffordshire NHS Foundation hospital. The report found that, over the duration of the incident, written patients complaints had identified the problems of neglect and poor care at the trust. Yet, deficiencies in complaint handling meant critical warning signs were missed, and numerous challenges in using patient complaint data to improve patient safety were highlighted. ${ }^{6}$

Healthcare organisations receive huge volumes of complaints (eg, over 100000 annually on hospital care in the NHS), and complaints can focus on diverse problems (from car parking to prescribing errors), describe different types of harm (eg, physical, emotional), have legal or malpractice implications, and have different underlying aims (eg, resolving dissatisfaction, creating change, preventing future issues). ${ }^{7-9}$ The level of standardisation of techniques used to analyse patient complaints is unclear, and there may be benefit in developing a standardised and 
reliable taxonomy for analysing and interpreting (in terms of patient safety) complaint data. To achieve this, we provide the first systematic review of empirical research on patient complaints. The aim of the review is to outline the practices used to code and analyse patient complaints, and to describe the types and prevalence of issues underlying patient complaints. Furthermore, through data synthesis, we develop a taxonomy for guiding and standardising the future analysis of patient complaints, with the purpose being to support research and practice on analysing and using information reported in letters of patient complaints.

\section{Patient letters of complaint}

Patient complaints usually refer to an 'expression of grievance' and 'dispute within a health care setting, ${ }^{10}$ They are often formal letters written to a healthcare organisation (or regulator) after a threshold of dissatisfaction with care has been crossed. ${ }^{11}$ Typically, complaints are made by patients or families. ${ }^{12}$ To resolve complaints, healthcare institutions usually create dialogue on the complaint, investigate it and reach a resolution for the individual patient (eg, apologise, reject, compensate). ${ }^{10}$ In considering how patient complaint data might be used to identify or reduce problems in patient safety, a number of distinguishing features of patient complaints require discussion.

First, patient complaints do not reflect a systematic investigation of failure; instead, they represent individual patient experiences. Complaints are unstandardised, although can report incidences of physical or mental harm. ${ }^{10}$ Second, patient complaints are often emotive. They can describe anger and distress, with the skills and attitudes of individual healthcare professionals being criticised. Third, patient complaints may not be put forward to improve healthcare provision, and thus may not explicitly highlight key learning points that could be used to improve healthcare services. Fourth, patient complaints often prioritise problems in interactions with healthcare staff, ${ }^{7} 13$ leading to a focus on subjective and less proceduralised aspects of treatment (eg, compassion and dignity), and how they interact with quality and safety. ${ }^{14}$ Fifth, patient complaints can be made without an awareness of the wider system pressures influencing care (eg, staff workloads), meaning they may not identify all of the contributory factors leading to a problem in care.

Nonetheless, patient complaint data can provide unique patient-centred insights into aspects of care that may not be easily captured through traditional quality and safety metrics (eg, dignity). Crucially, when patient complaints are considered at an aggregate level (eg, a hospital), they potentially indicate problematic trends in healthcare provision. Rigorous and systematic analytical procedures are essential if learning from patient complaints is to be facilitated. For example, in understanding the causes of adverse events, highly systematic and standardised investigatory techniques have been developed for analysing medical errors (eg, using human factors frameworks to identify causal factors ${ }^{15}$ ). These have facilitated the (i) generation of normative data on the frequency, nature and causes of adverse events; ${ }^{16}$ (ii) identification of challenges for different healthcare organisations/specialities; ${ }^{17}$ and (iii) interventions based on causal analyses. ${ }^{15}$ Similar rigour may be beneficial in extracting safety-related data from patient complaints. To contribute to future patient complaint research (and the patient safety literature more generally) our review has two aims.

The first aim is to systematically review quantitative articles reporting primary data on patient complaints in order to assess the nature and quality of the patient complaint research literature. We do this through:

1. Describing the characteristics of patient complaint studies.

2. Describing the methodologies used to collect and analyse patient complaint data.

The second aim is to synthesise quantitative data on the type and prevalence of issues that underlie patient complaints, and to propose a patient complaint coding taxonomy for guiding future research and practice. We do this through:

3. Reporting on and consolidating the issues identified as underlying patient complaints across the literature.

4. Sorting and thematically grouping the issues identified as underlying patient complaints in order to develop a datadriven patient complaint coding taxonomy.

\section{METHOD}

\section{Study selection}

This is the first systematic literature review on patient complaints in healthcare institutions. Accordingly, no protocol exists to guide the review, and so standard protocols for systematic review were applied. ${ }^{18}$ The starting eligibility criteria were articles reporting primary data on patient complaints in English. A search strategy to select relevant papers was then applied. Figure 1 describes the study selection procedure.

For stage 1, the search terminology was designed to limit the number of studies with the term 'complaint' in order that a relevant and manageable number of papers could be identified ('complaint' is a very common medical term). Studies were only identified if the word 'complaint' featured (or a common synonym, such as grievance or letter, or antonym such as satisfaction) alongside the words 'care', 'quality' or 'safety' in the title or abstract. This meant papers referring to the term 'complaint' only were not included.

For stage 2, the abstracts of articles with titles of relevance were inspected. For stage 3, the inclusion criteria identified articles with complaints submitted by patients on poor healthcare experiences (or representing third-parties), and excluded those where complaints were not initiated by patients or significant 


\begin{tabular}{|c|c|}
\hline $\begin{array}{l}\text { Stage 1: Initial search } \\
\text { Electronic Search: } \\
\text { Keywords: } \\
\text { Limitations: } \\
\text { Results: }\end{array}$ & $\begin{array}{l}\text { Pubmed, Science Direct and Web of Knowledge } \\
\text { Title and abstract contain car*[Title/abstract] OR quality [Title/abstract] OR safe* [Title/abstract] Or } \\
\text { satisfaction [Title/abstract] AND claim* [Title] OR complain* [Title] OR complim* [Title]OR grievanc }{ }^{*}\left[T_{i t l e]}\right. \\
\text { OR letter }{ }^{*} \text { Title] } \\
\text { Articles in English. } \\
3148 \text { Articles }\end{array}$ \\
\hline & $\downarrow$ \\
\hline $\begin{array}{l}\text { Stage 2: Screening of } \\
\text { Filter: } \\
\text { Filter: } \\
\text { Results: }\end{array}$ & $\begin{array}{l}\text { Titles examined for initial relevance } \\
\text { Relevance of abstract examined in order to assess whether data on patient complaints are presented in the } \\
\text { study } \\
106 \text { articles }\end{array}$ \\
\hline \multicolumn{2}{|r|}{$\downarrow$} \\
\hline $\begin{array}{l}\text { Stage 3: Initial Article } \\
\text { Inclusion criteria: } \\
\text { Exclusion criteria: } \\
\text { Hand search: } \\
\text { Results: }\end{array}$ & $\begin{array}{l}\text { Collects primary data on patient complaints, submitted by patients or third-parties on their behalf, in } \\
\text { healthcare organisations } \\
\text { Studies only refer to i) patient walkouts, ii) satisfaction questionnaires, iii) complaints made to non-healthcare } \\
\text { related organisations } \\
\text { Search of i) reference lists from included studies, and ii) articles citing identified articles in order to identify } \\
\text { further items of interest article type: } \\
77 \text { Articles }\end{array}$ \\
\hline \multicolumn{2}{|r|}{$\vee$} \\
\hline $\begin{array}{l}\text { Stage 4: Final article s } \\
\text { Filter: } \\
\text { Results: }\end{array}$ & $\begin{array}{l}\text { Stelection } \\
\text { complies filtered as to whether they contained quantitative data on the issues underlying and prompting patient } \\
59 \text { articles }\end{array}$ \\
\hline
\end{tabular}

Figure 1 Study selection procedure.

others (eg, elicited by researchers) were not submitted to healthcare organisations (or regulators), or reported poor healthcare experiences but not a specific complaint (eg, satisfaction surveys, patient walkouts). For all papers, the reference lists were hand-searched to identify other articles for potential inclusion. For stage 4 , papers providing quantitative data were selected. For stages 3 and 4, all papers were reviewed for inclusion independently by two psychologists (JR, TR). Inter-rater reliability for agreement was acceptable $(\kappa=0.71, \mathrm{p}<0.001)$, and where disagreement occurred a third psychologist adjudicated (AG).

\section{Data extraction and analysis}

The data were initially extracted by a single reviewer (JR), and then checked by a second reviewer (TR/AG). The process consisted of the following four phases.

\section{Phase I: descriptive data}

Data extraction. (1) Year published, (2) country, (3) healthcare environment, (4) number of patient complaints, (5) number of issues reported within a complaint, (6) characteristics of complainer, (7) gender of patient, (8) focus of complaints (medical or nursing staff) and (9) suggestion or implementation of interventions.

Data analysis. Trends on the year and country of research, the total number of complaints reported, issues identified, and characteristics of the patient, complainant and complainer.

Phase II: methodological data

Data extraction. (1) Medium of complaint capture, (2) whether multiple issues were identified in each complaint, (3) number of codes used to analyse complaints, (4) source of complaint coding frame and (5) who did the coding.

Data analysis. Trends on the use of complaint capture methodologies, methods of complaint analysis, use and development of theoretical models, and development or suggestion of interventions.

Phase III: issues raised in patient complaints

Data extraction. The number and types of issues reported as underlying patient complaints in all studies (ie, the causes of the complaint).

Data analysis. Aggregating the types and prevalence of issues found to underlie patient complaints. Across the studies selected for inclusion, there were 729 different complaint issue codes used. To facilitate the analysis and aggregation of data, it was necessary to synthesise the many issues used to account for complaints into semantically and conceptually parsimonious codes (eg, 'attitudes' were coded in 20 studies, using 15 different wording of 'attitude' codes). Figure 2 explains in detail the process for recoding. Recoding was conducted by a single reviewer (TR), 
2. Complaint issue codes were then recoded to develop parsimonious codes for aggregated analysis.
3. Complaint issue codes were then grouped together according to conceptual similarity.
4. Sub-category titles were generated, and the aggregate data on no. of complaint issues was calculated.
1. For each article, the complaint issue codes were extracted, alongside the number of issues associated with each code (and the total number of issues in each study). Issue codes were then sorted alphabetically so highly similar codes could be identified.

\begin{tabular}{|l|}
\hline Attitude 9 (244) \\
Attitude and behaviour 16 (215) \\
Attitude of staff 10 (100) \\
Attitude of healthcare professionals 37 (146] \\
Attitude or manner 2823 (23535) \\
Attitude problems 46 (207) \\
Attitude/behaviour 102 (586) \\
Attitudes of staff 21 (190) \\
Behaviour/attitude problems 91 (719) \\
Conduct and attitude 28 (223) \\
Delay in discharge from hospital 24 (360) \\
Discharge 28 (719) \\
Discharge / transfer unsuitable 59 (3418) \\
Discharge arrangements 110 (3418) \\
Discharge issues 27 (586) \\
Discharge issues 12 (646) \\
Discharge planning/follow up 7 (28) \\
Discharge procedure 3 (178) \\
Discharge process 23 (360) \\
Discharge 66 (1806) \\
Discharge/transfer $113(23535)$ \\
Discharge-inadequate $5(665)$ \\
Disputes about timing of discharge 8 (68) \\
Inadequate discharge 10 (188) \\
Poor staff attitude 32 (146) \\
Poor staff attitude $17(360)$ \\
Poor staff attitude 38 (188) \\
Premature discharge 142 (1171) \\
Problem with discharge 784 (15631) \\
Staff attitude 25 (109) \\
Staff attitude or social behaviour 128 (392) \\
Staff attitude/communication 77 (363) \\
Staff attitudes 7 (71) \\
Staff commitment 19 (646) \\
Unsatisfactory attitude of GP 48 (1000) \\
Wrong attitude 3 (178) \\
\end{tabular}

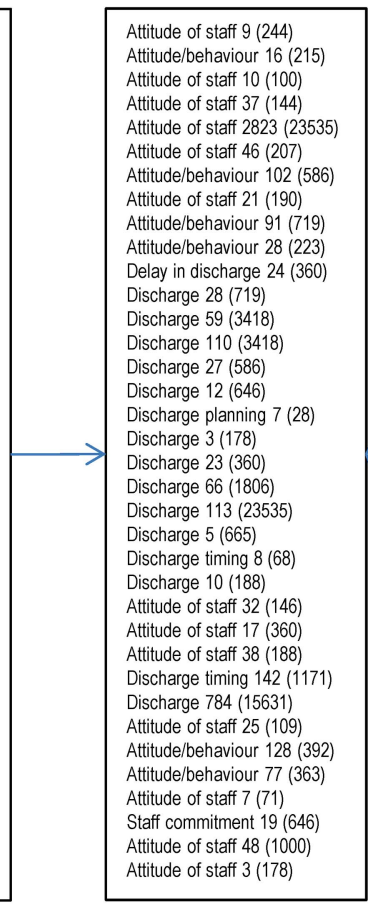

Figure 2 Illustration of the complaint issue recoding process

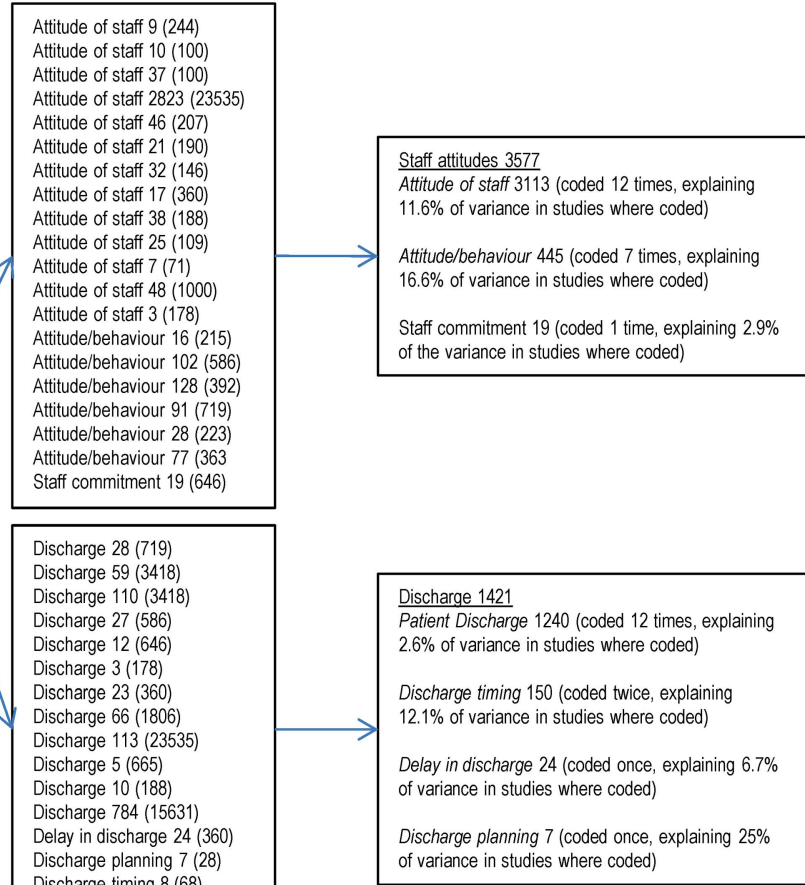

Discharge planning $7(28)$

Discharge timing 8 (68)

Discharge timing 142 (1171) and then checked for accuracy (JR/AG). The recoded issue codes were then grouped together conceptually and labelled (eg, into the 'subcategory' of 'attitude'), with the total number of issues associated with each issue code and subcategory being calculated.

Phase IV: developing a complaint coding taxonomy

The final stage of analysis involved the development of a complaint coding taxonomy. An inductive sorting exercise of the codes and subcategories identified in phase III was performed. Through a discursive process, the research team (TR, AG, and JR) created definitions for each subcategory (which captured the issues within each), and then thematically analysed the complaint subcategories into groupings that appeared conceptually similar (to create categories). The categories were then labelled to reflect the subcategories within them. The categories were then grouped together according to similarity in order to form 'domains' of patient complaint. These were labelled to reflect the categories within them and also to create links with relevant theories (ie, that might be used to understand or explain the content of complaints).

\section{RESULTS}

\section{Search results}

Fifty-nine publications were included. Articles were excluded for a range of factors, for example, not having extractable quantitative data, ${ }^{19} 20$ providing inexact data on complaint issues ${ }^{21}$ or focusing primarily on patient goals of complaining. ${ }^{9} 22$

Phase I: descriptive data

The articles identified in the review and their key characteristics are listed in table 1 . The earliest article was published in 1987, and research has steadily increased. Early research was conducted in the USA, UK and Australia, and more recently Taiwan, Netherlands, New Zealand, Canada, Iran, Sweden, Germany, Hong Kong, France and Singapore. The articles reported complaints from hospitals (51\%), emergency units (13\%), and a range of mixed and specific units (36\%). The number of complaints being reported on varied considerably (mean 1493, SD 3888, range 28-19 156, median 226).

The data for the complainant and complainer were only extractable in $61 \%$ of the studies. In the 36 studies (comprising 44211 complaints) reporting on the complainant, 64\% were patients and 26\% were family. In the 33 studies (comprising 36612 complaints) reporting the target of the complaint, $86 \%$ were medical staff, $6 \%$ were nursing staff and $8 \%$ were other. In all, 55\% of studies suggested an intervention and $17 \%$ implemented an intervention.

Phase II: methodological data

In the 43 articles reporting the medium for capturing patient complaints, $84 \%$ were written (with $61 \%$ of 
Table 1 Descriptive and methodological characteristics of identified article

\begin{tabular}{|c|c|c|c|c|c|c|c|c|c|}
\hline Authors & Year & Country & $\begin{array}{l}\text { Healthcare } \\
\text { environment }\end{array}$ & $\begin{array}{l}\text { No. of } \\
\text { complaints } \\
\text { reported }\end{array}$ & $\begin{array}{l}\text { System-level } \\
\text { intervention }\end{array}$ & $\begin{array}{l}\text { Single or } \\
\text { multiple } \\
\text { coding }\end{array}$ & $\begin{array}{l}\text { No. of } \\
\text { codes } \\
\text { used }\end{array}$ & $\begin{array}{l}\text { Source of } \\
\text { coding } \\
\text { frame }\end{array}$ & $\begin{array}{l}\text { Complaint } \\
\text { coded by }\end{array}$ \\
\hline Schwartz and Overton ${ }^{23}$ & 1987 & USA & Emergency & 244 & Suggested & Single & 12 & NR & Secretarial staff \\
\hline Chande et $a 2^{24}$ & 1991 & USA & Hospital & 176 & Suggested & Single & 11 & NR & Healthcare staff \\
\hline Owen ${ }^{25}$ & 1991 & UK & Primary care & 1000 & $N R^{*}$ & Single & 13 & NR & Author(s) \\
\hline Burstein and Fleisher ${ }^{26}$ & 1991 & USA & Hospital & 67 & Suggested & Multiple & 4 & NR & Unit manager \\
\hline Hunt and Glucksman ${ }^{27}$ & 1991 & UK & Emergency & 122 & Suggested & Multiple & 11 & NR & Author(s) \\
\hline $\begin{array}{l}\text { Donaldson and } \\
\text { Cavanagh }^{28}\end{array}$ & 1992 & UK & Hospital & 71 & Suggested & Multiple & 6 & NR & Healthcare staff \\
\hline Kadzombe and Coals ${ }^{29}$ & 1992 & UK & Emergency & 66 & NR & Multiple & 8 & NR & Unit manager \\
\hline Hanson et $a^{30}$ & 1994 & Australia & Child A\&E & 71 & Suggested & Single & 5 & NR & Parents \\
\hline Allsop 31 & 1994 & UK & Surgery & 110 & NR & Multiple & 6 & Literature & Author(s) \\
\hline Bark et al $l^{7}$ & 1994 & UK & Hospital & 491 & Suggested & Multiple & 9 & Literature & Author(s) \\
\hline $\begin{array}{l}\text { Lloyd-Bostock and } \\
\text { Mulcahy }{ }^{10} \dagger\end{array}$ & 1994 & UK & Hospital & 399 & Implemented & Multiple & 36 & Developed & Author(s) \\
\hline Nettleton and Harding ${ }^{32}$ & 1994 & UK & Multiple & 107 & NR & Multiple & 43 & NR & Author(s) \\
\hline Curka et $\left.a\right|^{\beta 3}$ & 1995 & USA & Emergency & 371 & Suggested & Single & 8 & NR & Unit manager \\
\hline Webb $^{34}$ & 1995 & UK & Hospital & 188 & Suggested & Single & 18 & NR & Healthcare staff \\
\hline Ingram and Roy ${ }^{35}$ & 1995 & UK & Psychiatric & 47 & NR & Multiple & 10 & NR & Author(s) \\
\hline $\begin{array}{l}\text { Mulholland and } \\
\text { Dawson }^{36}\end{array}$ & 1998 & NZ & Multiple & 146 & Suggested & Single & 5 & NR & Author(s) \\
\hline Mace $^{37}$ & 1998 & USA & Hospital & 28 & Implemented & Single & 5 & NR & Healthcare staff \\
\hline Lim et $\left.a\right|^{\beta 8}$ & 1998 & Singapore & Clinics & 226 & NR & Multiple & 38 & NR & Complaint staff \\
\hline Daniel et $a /^{39}$ & 1999 & Australia & Multiple & 290 & NR & Single & 12 & NR & Complaint staff \\
\hline Pichert et $a l^{40} \dagger$ & 1999 & USA & Hospital & 6419 & Implemented & Multiple & 15 & Developed & Complaint staff \\
\hline Allen et $\left.a\right|^{41} \ddagger$ & 2000 & USA & Hospital & 3984 & Implemented & Single & 5 & Developed & Patients \\
\hline Halperin ${ }^{42}$ & 2000 & USA & Multiple & 29 & NR & Single & 5 & NR & Complaint staff \\
\hline Anderson et $a /^{43}$ & 2000 & Australia & Hospital & 127 & NR & Multiple & 17 & NR & Patient advisor \\
\hline Pitarka-Carcani et al ${ }^{44}$ & 2000 & UK & Psychiatric & 100 & Implemented & Multiple & 23 & NR & NR \\
\hline Anderson et $a /^{45}$ & 2001 & Australia & Hospital & 1308 & NR & Multiple & 8 & NR & Patient advisor \\
\hline $\begin{array}{l}\text { Powers and } \\
\text { Bendall-Lyon }{ }^{46}\end{array}$ & 2002 & USA & Hospital & 211 & NR & Single & 5 & Literature & Complaint staff \\
\hline Taylor et a/ ${ }^{47}$ & 2002 & Australia & Emergency & 2419 & Suggested & Multiple & 43 & $N R$ & Complaint staff \\
\hline Colwell et al ${ }^{48}$ & 2003 & USA & Emergency & 286 & Suggested & Single & 10 & NR & Unit manager \\
\hline Choy et $a l^{49}$ & 2004 & Hong Kong & Hospital & 241 & Suggested & Multiple & 4 & NR & NR \\
\hline Taylor et $a 1^{50}$ & 2004 & Australia & Hospital & 19156 & Suggested & Multiple & 7 & $N R$ & Complaint staff \\
\hline Wofford et $\left.a\right|^{51}$ & 2004 & USA & Hospital & 222 & Suggested & Multiple & 7 & Developed & Author(s) \\
\hline Stelfox et $\left.a\right|^{52}$ & 2005 & USA & Hospital & 483 & NR & Single & 2 & NR & NR \\
\hline Friele and Sluijs ${ }^{53}$ & 2006 & Netherlands & Hospital & 424 & NR & Multiple & 4 & Literature & Patients \\
\hline Chavan et $\left.a\right|^{54}$ & 2007 & UK & Eye infirmary & 94 & Implemented & Single & 6 & NR & Complaint staff \\
\hline Cowan and Wilson ${ }^{55} \dagger$ & 2007 & UK & Surgery & 526 & Suggested & Single & 6 & NR & Healthcare staff \\
\hline Kline et $\left.a\right|^{56}$ & 2007 & Canada & Hospital & 586 & Suggested & Single & 11 & NR & Complaint staff \\
\hline Saravanan et a ${ }^{57}$ & 2007 & UK & Hospital & 100 & Suggested & Single & 6 & NR & NR \\
\hline Siyambalapitiya et $a l^{58} \dagger$ & 2007 & UK & Hospital & 183 & Suggested & Single & 6 & NR & Complaint staff \\
\hline Natangelo ${ }^{59}$ & 2007 & Italy & Hospital & 151 & Suggested & Multiple & 6 & Literature & Healthcare staff \\
\hline Wong et $a l^{60}$ & 2007 & Singapore & Emergency & 175 & Suggested & Multiple & 16 & NR & Author(s) \\
\hline Haw et al ${ }^{61}$ & 2008 & UK & Psychiatric & 392 & Implemented & Single & 15 & NR & Complaint staff \\
\hline Friele et $a l^{62}$ & 2008 & Netherlands & Hospital & 279 & Suggested & Multiple & 5 & Literature & Patients \\
\hline Montini et al ${ }^{63}$ & 2008 & USA & Hospital & 1216 & Implemented & Multiple & 22 & Developed & Author(s) \\
\hline Giugliani et $a /^{64}$ & 2009 & France & Hospital & 164 & NR & Single & 6 & NR & Healthcare staff \\
\hline Parry and Hewage ${ }^{65}$ & 2009 & Australia & Hospital & 101 & Suggested & Single & 4 & NR & Complaint staff \\
\hline Jangland et $a l^{13}$ & 2009 & Sweden & Hospital & 105 & Implemented & Multiple & 14 & Literature & Author(s) \\
\hline Levtzion-Korach et a ${ }^{3}$ & 2010 & USA & Hospital & 4722 & Suggested & Multiple & 8 & Literature & Author(s) \\
\hline
\end{tabular}


Table 1 Continued

\begin{tabular}{|c|c|c|c|c|c|c|c|c|c|}
\hline Authors & Year & Country & $\begin{array}{l}\text { Healthcare } \\
\text { environment }\end{array}$ & $\begin{array}{l}\text { No. of } \\
\text { complaints } \\
\text { reported }\end{array}$ & $\begin{array}{l}\text { System-level } \\
\text { intervention }\end{array}$ & $\begin{array}{l}\text { Single or } \\
\text { multiple } \\
\text { coding }\end{array}$ & $\begin{array}{l}\text { No. of } \\
\text { codes } \\
\text { used }\end{array}$ & $\begin{array}{l}\text { Source of } \\
\text { coding } \\
\text { frame }\end{array}$ & $\begin{array}{l}\text { Complaint } \\
\text { coded by }\end{array}$ \\
\hline Moghadam et al ${ }^{66}$ & 2010 & Iran & Cardiac & 1642 & Implemented & Single & 10 & Developed & Complaint staff \\
\hline Stimson et $a /^{67}$ & 2010 & USA & Hospital & 1516 & Suggested & Single & 5 & Literature & Complaint staff \\
\hline Hsieh $^{68}$ & 2010 & Taiwan & Hospital & 59 & Suggested & Multiple & 8 & Literature & Social worker \\
\hline McGregor et $a{ }^{69} \S$ & 2011 & Canada & Nursing home & 629 & $N R$ & Single & 28 & $N R$ & Complaint staff \\
\hline Gogos et $a l^{70} \S$ & 2011 & Australia & Multiple & 481 & NR & Multiple & 5 & Literature & Healthcare staff \\
\hline Hsieh $^{8}$ & 2012 & Taiwan & Hospital & 665 & Suggested & Single & 38 & $N R$ & Social worker \\
\hline Källberg et al ${ }^{71}$ & 2012 & Sweden & Emergency & 1778 & Suggested & Single & 10 & Developed & Author(s) \\
\hline Sachdeo et al ${ }^{72}$ & 2012 & USA & Dental clinic & 214 & Suggested & Single & 5 & NR & Author(s) \\
\hline Schnitzer et al ${ }^{73} \dagger$ & 2012 & Germany & Multiple & 13505 & NR & Single & 4 & $N R$ & Author(s) \\
\hline Mann et $a l^{74}$ & 2012 & UK & Surgery & 113 & Suggested & Multiple & 15 & Literature & Healthcare staff \\
\hline van Mook et $a l^{75}$ & 2012 & Netherlands & Multiple & 137 & Suggested & Multiple & 29 & Literature & Author(s) \\
\hline Bismark et $a l^{76}$ & 2013 & Australia & Multiple & 18907 & Suggested & Multiple & 20 & Literature & Author(s) \\
\hline
\end{tabular}

${ }^{*} \mathrm{NR}$, not retrievable.

tDiscrepancy between the total number of complaints reported and the tally of the individual complaints we managed to extract.

FImperfect data extraction due to the study reporting rounded percentages.

$\S$ Study analyses a subset of 'substantiated' complaints.

A\&E, Accident and Emergency.

these supplemented with verbal data) and $12 \%$ were questionnaire-based. In all, 49\% of articles coded each complaint as pertaining to a single issue, while $51 \%$ coded a complaint as raising multiple issues. Of those with extractable data, there were 1.49 issues per complaint (SD 0.544, range 1.05-3.19, median 1.56).

Thirteen articles (24\%) applied a theoretical framework from the literature on complaints, while seven $(12 \%)$ developed their own framework. For example, a framework developed by Lloyd-Bostock and Mulcahy ${ }^{10}$ viewed complaints as social episodes, and focused on detailed 'nonclinical' and 'clinical' issues. In some cases, ${ }^{53} 6277$ complaint coding was influenced by the consumer complaint literature. ${ }^{78}$ Montini et $a l^{63}$ developed arguably the most rigorous coding frame through an analysis of 1216 complaints using seven complaint codes identified in eight articles on malpractice and complaints. Some studies relied on pre-existing institutional frameworks to analyse complaints, ${ }^{74}$ or developed their own frameworks based on initial coding and qualitative work. ${ }^{13} \quad 40 \quad 68 \quad 71$ Overall, 38 articles (64\%) did not provide a clear rationale for the codes used.

Finally, of the 55 articles reporting who did the coding of complaints, $33 \%$ were done by the authors, $27 \%$ were done by a complaints department, $16 \%$ were done by healthcare staff, and $10 \%$ were done by patients or their advocates. No study graded complaints by severity or reported inter-rater reliability in coding complaints.

Phase III: issues raised in patient complaints

Across the 59 papers, 729 issue codes were used to code the 88069 complaints, and the number of issues totalled 113551 (ranging from $29^{42}$ to $26785^{47}$ ). The number of issue codes used to analyse complaints varied from $4^{65}$ to $43,{ }^{47}$ with a mean of 12.2 (SD 10.3 ) codes. After collapsing issue codes on the basis of similarity (see figure 2), 205 unique issue codes remained. Of these, 103 involved the amalgamation of data from at least two studies. Overall, 84 issue codes were classified as miscellaneous (accounting for 2388 issues) as they referred to codes that were unclassifiable (eg, 'other').

The 205 issue codes were collapsed into 29 subcategories. Figure 3 reports on the issue codes (and their overarching subcategories), the number of times they were used, the number of issues explained by them and the proportion of complaint issues they explain in studies where they were reported. At the subcategory level, issues most frequently related to codes on 'treatment' (17 716) and 'communication' (15 621) and the fewest to 'staffing' (47). In terms of the number of 'codings' for each subcategory (ie, how many times a code was used across the literature), the most were for 'delays' (55) and 'environment' (40), and the least for 'staffing' (5). In terms of the proportion of patient complaint issues explained by subcategories of issue codes (in studies where codes were used), the most were for 'treatment' (22.1\%) and 'communication' $(16.8 \%)$ and the least for 'discrimination' $(0.5 \%)$.

Phase IV: developing a complaint coding taxonomy

To integrate and structure the complaint issue codes and subcategories in figure 3, a final sorting exercise was performed. Subcategories were defined to reflect the issues within each and to be made distinct from one another (ie, to reduce overlap between constructs). They were then sorted into seven categories, and these were inductively sorted into three domains (figure 4). 


\begin{tabular}{|c|c|c|c|c|c|c|c|c|c|c|c|}
\hline $\begin{array}{c}\text { Complaint issue sub- } \\
\text { categories, and underlying } \\
\text { issue codes }\end{array}$ & & $\begin{array}{l}\text { of codi } \\
\text { ues } 1 \% \\
\text { lained (v }\end{array}$ & $\begin{array}{l}\text { I No. of } \\
\text { a issues } \\
\text { re coded) }\end{array}$ & $\begin{array}{l}\text { Prescribing } \\
\text { Medication omission }\end{array}$ & $\begin{array}{l}7 \\
1\end{array}$ & $\begin{array}{l}102 \\
1\end{array}$ & $\begin{array}{l}5.6 \% \\
0.7 \%\end{array}$ & $\begin{array}{l}\text { Visiting times } \\
\text { Miscellaneous }\end{array}$ & $\underline{84}$ & $\underline{2388}$ & $\begin{array}{l}0.5 \% \\
\underline{6.3 \%} \\
\end{array}$ \\
\hline$x^{2}$ & & & & Respect, dignity, and caring & $\underline{36}$ & $\underline{4378}$ & $13.9 \%$ & & & & \\
\hline & $\underline{34}$ & $\underline{17716}$ & $\underline{22.1 \%}$ & Lack of concern & 2 & 1621 & $9.5 \%$ & Incorrect information & $\underline{18}$ & 1968 & $\underline{6.2 \%}$ \\
\hline Poor treatment & 13 & 16605 & $22.3 \%$ & Rude behaviour & 10 & 1095 & $18.8 \%$ & Inadequate information & 7 & 1455 & $5.6 \%$ \\
\hline Inadequate/inappropriate treat. & 13 & 543 & $7.6 \%$ & Respect and Caring & 1 & 741 & $18.6 \%$ & Incomplete/inaccurate info & 7 & 466 & $8.3 \%$ \\
\hline Medical treatment & 1 & 184 & $29.5 \%$ & Humaneness & 2 & 275 & $1.7 \%$ & Conflicting information & 3 & 38 & $2.6 \%$ \\
\hline Complication not explained & 1 & 154 & $70.6 \%$ & Uncaring & 2 & 186 & $4.9 \%$ & Misinformation & 1 & 9 & $3.4 \%$ \\
\hline Incorrect treatment & 1 & 137 & $11.7 \%$ & Insensitive & 7 & 188 & $6.1 \%$ & & & & \\
\hline Clinical treatment & 1 & 31 & $31.0 \%$ & Disrespect, & 4 & 163 & $12.4 \%$ & Discharge & $\underline{16}$ & 1421 & $\underline{2.9 \%}$ \\
\hline Non-performance of treatment & 1 & 23 & $14.0 \%$ & Insufficient empathy & 1 & 30 & $12.6 \%$ & Patient discharge & $\overline{12}$ & $\overline{1240}$ & $2.6 \%$ \\
\hline Poor explanation of treatment & 1 & 20 & $29.4 \%$ & Insufficient acknowledgement & 1 & 26 & $10.9 \%$ & Discharge timing & 2 & 150 & $12.1 \%$ \\
\hline Poor treatment plan & 1 & 18 & $26.5 \%$ & Lack of privacy & 2 & 17 & $3.0 \%$ & Delay in discharge & 1 & 24 & $6.7 \%$ \\
\hline Unsuccessful treatment & 1 & 1 & $0.6 \%$ & $\begin{array}{l}\text { Undignified service } \\
\text { Neglect }\end{array}$ & 2 & 17 & $0.5 \%$ & Discharge planning & 1 & 7 & $25.0 \%$ \\
\hline Communication & $\underline{33}$ & 15621 & $16.8 \%$ & $\begin{array}{l}\text { Neglect } \\
\text { Insufficient. emotional support }\end{array}$ & $\begin{array}{l}1 \\
1\end{array}$ & $\begin{array}{l}14 \\
5\end{array}$ & $\begin{array}{l}2.2 \% \\
2.1 \%\end{array}$ & Safety incidents & $\underline{20}$ & 1052 & $6.1 \%$ \\
\hline Poor communication & 33 & $\overline{15621}$ & $\overline{16.8 \%}$ & & & & & Medical error & $\overline{4}$ & 425 & $\frac{. .170}{4.7 \%}$ \\
\hline & & & & Staff attitudes & $\underline{20}$ & $\underline{3577}$ & $13.6 \%$ & Surgical/medical complication & 4 & 203 & $12.6 \%$ \\
\hline Access & $\underline{38}$ & $\underline{11470}$ & $\underline{14.8 \%}$ & Attitude of staff & 12 & 3113 & $11.6 \%$ & Adverse event & 4 & 169 & $5.8 \%$ \\
\hline$\overline{\text { Access to services }}$ & $\overline{13}$ & $\overline{9870}$ & $13.0 \%$ & Attitude/behaviour & 7 & 445 & $16.6 \%$ & Organisational incidents & 1 & 156 & $26.9 \%$ \\
\hline Admission/appointment issues & 2 & 575 & $30.8 \%$ & Staff commitment & 1 & 19 & $2.9 \%$ & Equipment failure & 5 & 79 & $2.7 \%$ \\
\hline Appointments & 8 & 459 & $2.7 \%$ & & & & & Safety problems & 1 & 17 & $0.9 \%$ \\
\hline Access/availability & 4 & 370 & $19.1 \%$ & Skills and conduct & $\underline{35}$ & $\underline{3544}$ & $15 \%$ & Patient death & 1 & 3 & $1.2 \%$ \\
\hline Not admitted & 3 & 101 & $2.0 \%$ & Staff Behaviour & $\overline{2}$ & 1529 & $19.6 \%$ & & & & \\
\hline No consultation & 1 & 24 & $0.7 \%$ & Clinical judgement & 1 & 814 & $12.3 \%$ & Abuse & $\underline{18}$ & $\underline{815}$ & $2.5 \%$ \\
\hline Refusal to treat & 1 & 21 & $0.6 \%$ & Unprofessional conduct & 9 & 304 & $5.0 \%$ & Sexual behaviour & 3 & 651 & $2.7 \%$ \\
\hline Busy & 1 & 17 & $0.5 \%$ & Medical staff conduct & 1 & 288 & $49.7 \%$ & Patient aggression & 1 & 35 & $8.9 \%$ \\
\hline Registration & 2 & 16 & $3.0 \%$ & Incompetence/negligence & 1 & 103 & $35.5 \%$ & Physical abuse & 2 & 32 & $0.8 \%$ \\
\hline Refused visit & 1 & 14 & $8.4 \%$ & Misapplication of clinical skills & 1 & 98 & $45.6 \%$ & Staff to resident abuse & 2 & 20 & $2.0 \%$ \\
\hline Doctor availability & 1 & 2 & $1.2 \%$ & Technical skills & 2 & 77 & $8.3 \%$ & Emotional/verbal abuse & 1 & 15 & $2.4 \%$ \\
\hline Admissions & 1 & 1 & $0.5 \%$ & Ignorance of care standards & 1 & 68 & $4.1 \%$ & Physical/sexual abuse & 1 & 15 & $5.2 \%$ \\
\hline & & & & Poor staff team working & 4 & 64 & $5.0 \%$ & Excessive force & 1 & 11 & $3.8 \%$ \\
\hline Bureaucracy & $\underline{23}$ & $\underline{9647}$ & $11.9 \%$ & Nursing conduct & 1 & 61 & $9.8 \%$ & Overzealous evaluation & 1 & 10 & $5.7 \%$ \\
\hline Administration & $\overline{11}$ & $\overline{5664}$ & $\overline{10.8 \%}$ & Unsuitable/insufficient staff & 1 & 43 & $6.8 \%$ & Intimidation & 1 & 9 & $1.4 \%$ \\
\hline Policies & 3 & 3240 & $18.5 \%$ & Poor professional skills & 2 & 37 & $1.0 \%$ & Financial abuse & 1 & 5 & $0.8 \%$ \\
\hline Organisation of care & 3 & 469 & $15.4 \%$ & Driving skills & 1 & 14 & $4.9 \%$ & Provider assaulted patient & 1 & 5 & $0.3 \%$ \\
\hline Grievance handling & 1 & 213 & $0.9 \%$ & Inappropriate staff conduct & 1 & 13 & $2.1 \%$ & Abuse & 1 & 4 & $0.7 \%$ \\
\hline Documentation issues & 3 & 37 & $1.4 \%$ & Unqualified staff & 1 & 10 & $1.6 \%$ & Resident to resident abuse & 1 & 2 & $0.3 \%$ \\
\hline Political & 1 & 14 & $1.9 \%$ & Poor clinical leadership & 1 & 7 & $1.1 \%$ & Alleged abuse & 1 & 1 & $0.2 \%$ \\
\hline Procedures & 1 & 10 & $9.9 \%$ & Abuse of position & 1 & 5 & $2.1 \%$ & & & & \\
\hline & & & & Irresponsible staff & 1 & 4 & $1.1 \%$ & Consent & $\underline{6}$ & $\underline{655}$ & $2.5 \%$ \\
\hline Finance and billing & $\underline{31}$ & $\underline{6418}$ & $7.7 \%$ & Inappropriate attendance & 1 & 2 & $1.6 \%$ & Problems in consent & 5 & 635 & $2.5 \%$ \\
\hline Billing & 16 & 4869 & $10.9 \%$ & Inexperienced staff & 1 & 2 & $0.5 \%$ & Coercion & 1 & 20 & $1.1 \%$ \\
\hline Costs & 3 & 973 & $3.6 \%$ & Professional discipline & 1 & 1 & $0.2 \%$ & & & & \\
\hline Financial & 9 & 557 & $4.7 \%$ & & & & & Confidentiality & $\underline{9}$ & $\underline{499}$ & $1.6 \%$ \\
\hline Parking fee & 2 & 12 & $0.9 \%$ & Delays & $\underline{55}$ & $\underline{3152}$ & $\underline{8.7 \%}$ & Patient confidentiality & $\overline{9}$ & $\overline{499}$ & $\overline{1.6 \%}$ \\
\hline Business practice & 1 & 7 & $8.0 \%$ & Waiting Time & 30 & 1640 & $6.2 \%$ & & & & \\
\hline & & & & Clinical delay & 2 & 414 & $11.5 \%$ & Examinations & $\underline{18}$ & $\underline{333}$ & $\underline{6.8 \%}$ \\
\hline Quality care & $\underline{41}$ & $\underline{5228}$ & $8.9 \%$ & Delay in treatment & 6 & 308 & $10.5 \%$ & Patient examination & $\overline{10}$ & $\overline{172}$ & $6.2 \%$ \\
\hline Care/treatment & 6 & $\overline{1919}$ & $9.9 \%$ & Timeliness & 3 & 304 & $6.6 \%$ & Failure to investigate & 3 & 120 & $5.0 \%$ \\
\hline Care quality & 9 & 1845 & $24.3 \%$ & Delay in diagnosis & 4 & 217 & $11.5 \%$ & Poor investigation & 2 & 22 & $4.4 \%$ \\
\hline Rough treatment & 3 & 502 & $1.9 \%$ & Delay in admission & 3 & 112 & $3.0 \%$ & Tests & 1 & 15 & $2.1 \%$ \\
\hline Nursing care & 6 & 233 & $3.5 \%$ & Failure/delay in visit & 4 & 106 & $4.8 \%$ & Unnecessary examination & 1 & 2 & $0.5 \%$ \\
\hline Clinical Services & 1 & 220 & $60.6 \%$ & Delay of ED admission & 1 & 43 & $4.3 \%$ & Not taking radiographs & 1 & 2 & $0.8 \%$ \\
\hline Medical care & 5 & 208 & $13.6 \%$ & Delays in A\&E & 1 & 5 & $2.7 \%$ & & & & \\
\hline Lack of care & 4 & 137 & $8.2 \%$ & Delays in outpatient dept & 1 & 3 & $1.6 \%$ & Communication breakdown & 10 & $\underline{284}$ & $5.5 \%$ \\
\hline Pain management & 2 & 78 & $3.2 \%$ & & & & & Communication breakdowns & 2 & 200 & $9.2 \%$ \\
\hline Absence of due care & 1 & 32 & $21.9 \%$ & Patient-staff dialogue & $\underline{20}$ & $\underline{2810}$ & $\underline{8.0 \%}$ & Lack of communication & 2 & 48 & $5.1 \%$ \\
\hline Hospital acquired infection & 2 & 23 & $4.4 \%$ & Doctor-patient relationship & 2 & 1310 & $9.6 \%$ & Poor conversation skills & 1 & 12 & $5.0 \%$ \\
\hline Health service care & 1 & 18 & $17.8 \%$ & Not listening & 2 & 624 & $3.8 \%$ & Language problems & 2 & 9 & $2.0 \%$ \\
\hline Clinical complaints & 1 & 13 & $13.8 \%$ & Interpersonal communication & 3 & 365 & $21.1 \%$ & Miscommunication & 1 & 9 & $3.4 \%$ \\
\hline & & & & Blamed patient & 1 & 302 & $1.9 \%$ & Inadequate communication & 1 & 5 & $3.4 \%$ \\
\hline Error in diagnosis & $\underline{29}$ & $\underline{5220}$ & $10.0 \%$ & Explanations to patients & 5 & 59 & $2.5 \%$ & Communication delay & 1 & 1 & $0.7 \%$ \\
\hline Diagnosis & 8 & 4335 & $9.5 \%$ & Disagree care expectations & 1 & 51 & $19.2 \%$ & & & & \\
\hline Misdiagnosis & 11 & 345 & $5.1 \%$ & Distrust & 1 & 40 & $15.0 \%$ & Patient Joumey & 10 & 137 & $3.2 \%$ \\
\hline Missed diagnosis & 3 & 274 & $20.6 \%$ & Unnecessary comment & 1 & 18 & $4.9 \%$ & Lack of follow up & 3 & 85 & $3.2 \%$ \\
\hline Diagnostic procedures & 1 & 189 & $10.6 \%$ & Conflict management & 1 & 15 & $3.8 \%$ & Patient flow issues & 4 & 38 & $2.6 \%$ \\
\hline Lack of or mis-diagnosis & 1 & 43 & $14.8 \%$ & Insufficient participation & 1 & 13 & $5.5 \%$ & Poor future planning & 3 & 14 & $0.9 \%$ \\
\hline Triage & 2 & 20 & $1.0 \%$ & Decision sharing & 2 & 13 & $1.5 \%$ & & & & \\
\hline Disputes over diagnosis & 1 & 7 & $10.3 \%$ & & & & & Discrimination & 8 & 141 & $0.5 \%$ \\
\hline Slow diagnosis & 1 & 6 & $20.7 \%$ & Patient rights & $\underline{7}$ & $\underline{2242}$ & $\underline{6.6 \%}$ & Discrimination against patient & $\overline{6}$ & 136 & $0.5 \%$ \\
\hline Contradictory diagnoses & 1 & 1 & $0.6 \%$ & Rights & $\overline{4}$ & $\overline{2212}$ & $\overline{7.1 \%}$ & Prejudice & 2 & 5 & $1.1 \%$ \\
\hline & & & & Breach of rights & 3 & 30 & $0.9 \%$ & & & & \\
\hline Environment & $\underline{40}$ & $\underline{5125}$ & $5.9 \%$ & & & & & Referrals & $\underline{7}$ & $\underline{125}$ & $2.1 \%$ \\
\hline Poor environment & 21 & 2952 & $5.0 \%$ & $\underline{\text { Senvice issues }}$ & $\underline{35}$ & $\underline{2425}$ & $\underline{5.3 \%}$ & Delay in referral & 2 & 53 & $3.5 \%$ \\
\hline Accommodation & 3 & 1770 & $37.5 \%$ & Medical records & 9 & $\overline{1429}$ & $4.7 \%$ & Referral & 3 & 50 & $5.6 \%$ \\
\hline Hygiene & 4 & 234 & $0.9 \%$ & Services & 2 & 335 & $4.2 \%$ & Refusal to refer & 2 & 22 & $0.6 \%$ \\
\hline Food & 6 & 72 & $3.4 \%$ & No service & 1 & 234 & $6.8 \%$ & & & & \\
\hline Sanitation & 1 & 28 & $4.5 \%$ & Transport & 6 & 145 & $7.7 \%$ & Staffing & $\underline{5}$ & $\underline{47}$ & $2.4 \%$ \\
\hline Amenities & 2 & 26 & $15.3 \%$ & Unreturned calls & 1 & 69 & $3.8 \%$ & Staffing & 2 & 25 & $2.3 \%$ \\
\hline Other patients & 1 & 20 & $5.1 \%$ & Telephone Consultation & 1 & 64 & $28.3 \%$ & Insufficient nursing & 1 & 15 & $6.3 \%$ \\
\hline Availability of linens/blankets & 1 & 13 & $1.8 \%$ & Wrong hospital & 1 & 30 & $8.1 \%$ & Insufficient practical support & 1 & 6 & $2.5 \%$ \\
\hline Disability accommodation & 1 & 10 & $0.6 \%$ & Reception & 4 & 19 & $1.5 \%$ & Over licensed capacity & 1 & 1 & $0.2 \%$ \\
\hline & & & & System malfunctions & 1 & 17 & $2.6 \%$ & & & & \\
\hline Medication errors & $\underline{23}$ & $\underline{5113}$ & $10.9 \%$ & Cancellations & 1 & 14 & $1.9 \%$ & Total & $\underline{729}$ & $\underline{113551}$ & \\
\hline Restriction of drugs & $\overline{1}$ & $\overline{3214}$ & $23.8 \%$ & Patient education & 2 & 7 & $0.5 \%$ & & & & \\
\hline Medication & 4 & 1607 & $6.1 \%$ & Parking & 4 & 58 & $3.0 \%$ & & & & \\
\hline Medication errors & 10 & 189 & $3.0 \%$ & Ward allocation & 1 & 3 & $0.4 \%$ & & & & \\
\hline
\end{tabular}

Figure 3 List of complaint issue codes and subcategories.

Figure 4 was developed in a bottom-up manner and develops a tri-level system of complaint coding. At the top-level, it distinguishes among the domains of 'clinical', 'management' and 'relationship' for analysing patient complaints. The 'clinical' domain (33.7\% of complaint issues) pertains to patient 


\begin{tabular}{|c|c|c|c|}
\hline Domains & Categories & Sub-categories & \\
\hline \multirow{8}{*}{ Clinical } & \multirow{4}{*}{ Quality } & Examinations: & Inadequate patient examination by clinical staff \\
\hline & & Patient Journey: & Problems in the coordination of treatment in different services by clinical staff \\
\hline & & Quality of care: & Substandard clinical/nursing care \\
\hline & & Treatment: & Poor, or unsuccessful, clinical treatment \\
\hline & \multirow{4}{*}{ Safety } & Errors in diagnosis: & Erroneous, missed, or slow clinical diagnosis \\
\hline & & Medication errors: & Errors in prescribing or administering medications \\
\hline & & Safety incidents: & Events or complications that threatened the safety of patients \\
\hline & & Skills and conduct: & Deficiencies in the technical and non-technical skills of staff that compromise safety \\
\hline \multirow{9}{*}{ Management } & \multirow{5}{*}{ Institutional issues } & Bureaucracy: & Problems with administrative policies and procedures \\
\hline & & Environment: & Poor accommodation, hygiene, or food \\
\hline & & Finance and billing: & Healthcare-associated costs, or the billing process \\
\hline & & Service issues: & Problems with hospital services for supporting patients \\
\hline & & Staffing and resources: & Inadequate hospital staffing and resource levels \\
\hline & \multirow{4}{*}{ Timing and access } & Access and admission: & Lack of access to services or staff \\
\hline & & Delays: & Delays in admissions or access to treatment \\
\hline & & Discharge: & Early, late, or unplanned discharge from the hospital \\
\hline & & Referrals: & Problems in being referred to a healthcare service \\
\hline \multirow{8}{*}{ Relationships } & \multirow{3}{*}{ Communication } & Communication breakdown: & Inadequate, delayed, or absent communication with patients \\
\hline & & Incorrect Information: & Communication of wrong, inadequate, or conflicting information to patients \\
\hline & & Patient-staff dialogue: & Not listening to patients, lack of shared decision-making, and conflict \\
\hline & Humaneness/caring & $\begin{array}{l}\text { Respect, dignity, and caring: } \\
\text { Staff attitudes: }\end{array}$ & $\begin{array}{l}\text { Rude, disrespectful, or insensitive behaviours to patients } \\
\text { Poor attitudes towards patients or their families }\end{array}$ \\
\hline & \multirow{4}{*}{ Patient rights } & Abuse: & Physical, sexual, or emotional abuse of patients \\
\hline & & Confidentiality: & Breaches of patient confidentiality \\
\hline & & Consent: & Coercing or failing to obtain patient consent \\
\hline & & Discrimination: & Discrimination against patients \\
\hline
\end{tabular}

Figure 4 Patient complaint taxonomy.

reports on poor quality care (23 414 issues) and safety incidents (14929 issues), and conceptually these categories relate to the human factors and safety literature. $^{79} 80$ The 'management' domain (35.1\%) pertains to problems in waiting times/access to care (16 043 issues) and institutional management (23 787 issues), and may be understood in terms of literatures on service and organisational management. ${ }^{81-83}$ Finally, the 'relationship' domain (29.1\%) considers patient complaints on interactions and experiences of healthcare professionals. These refer to communicatory acts between patients and staff (20 683 issues), the humaneness and caring nature of staff (7955 issues), and failures to fulfil basic patient rights (4352 issues). These categories reflect psychology literatures on communication and dialogue, ${ }^{84}$ compassion and emotional care, ${ }^{85} 86$ and workplace rule violations. ${ }^{87-89}$ It is notable that for the subcategories of 'communication' and 'patient rights' reported in figure 3, these were incorporated into the 'category' level as they did not provide insight at the subcategory level (ie, they were umbrella terms and highly general), and the subcategory of 'miscellaneous' was not included (meaning the taxonomy has 26 subcategories).

Finally, using the taxonomy to reflect on the patient complaint studies, it was found that there were different distributions of complaints between studies that coded complaints as having a single (29 studies) or multiple (30) issues. Single coded complaints reported more 'institutional issues' $(35.6 \%$ vs $16.3 \%)$ and fewer problems in 'communication' (14.3\% vs $19.4 \%)$ and 'quality' ( $12.5 \%$ vs $23.1 \%)$.

\section{DISCUSSION}

Investigations of patient complaints are increasing, both in total number and countries represented. Analyses of patient complaints serve two key functions within healthcare organisations. First, they allow for the concerns of specific patients to be met, for redress to occur and for solutions to be designed for solving case-specific safety problems. Second, they can provide insight into system-wide problems in patient care and allow for comparisons between healthcare organisations. The latter requires aggregate analyses of patient complaints in order that patient complaint data can be used to identify safety and quality issues within healthcare systems or conditions (eg, management problems) that increase the likelihood of poor care. Of the 59 papers reviewed in this study, patients were found to complain almost equally on the domains of 'clinical', 'management' and 'relationships'. Some institutional factors appear more specific to certain healthcare systems (eg, finance and billing in the USA), while others are more generic (eg, delays). Overall, 39\% of complaint issues focus on two of the seven categories, 'communication' and 'treatment', and a further $13 \%$ of complaint issues relate to 'safety'.

\section{Methodological issues in coding patient complaints}

The review highlights a lack of standardised codification or analytical techniques for analysing complaints. System-wide analyses of patient complaint data will remain difficult unless the following inconsistencies are addressed.

First, there is little standardisation of the procedures for analysing complaints (eg, training) or the purpose of data collection (ie, for redressing individual complaints or system-wide issues). In addition, the process of who handles and codes complaints differs considerably between studies, with little data on coding reliability. Second, the process of complaint coding varies widely, with different codes and frameworks (or none at all) being used. Research studies code and mix data at both the category and subcategory level, and often 
do not apply a theoretical framework. Third, the codes used sometimes pertain to the stage of healthcare (ie, referral, examination, treatment) and sometimes to problems at a more general level (ie, safety incident, delays, staff attitudes). Fourth, in some cases, patient complaints are treated as representing one issue, and in others multiple issues (51\% of studies). Multiple coding is more rigorous, and reveals patient complaints to focus on several interconnected aspects of healthcare. Fifth, where multiple issues are coded, complaint studies do not link issues within complaints together (eg, staff attitudes as precursors to adverse events), meaning complaints about one issue (eg, communication) cannot be linked to another (eg, clinical harm). Sixth, complaint studies do not typically collect data on the reliability of complaint analyses or on the severity of patient complaint (standard within adverse event studies). ${ }^{90}$ Seventh, because patient complaint letters are usually focused on the initial communique to healthcare providers, additional information found through follow-up studies (eg, interviews) are rarely incorporated into analyses. Finally, safety issues were only coded in $60 \%$ of the studies, yet where coded they often accounted for a considerable proportion of complaints, indicating the potential utility of analysing patient complaints to identify safety-specific data.

\section{Patient complaint coding taxonomy}

To overcome some of the issues highlighted above, we have developed a three-level complaint coding taxonomy (figure 4). It is designed to provide a standardised and comprehensive system for aiding researchers and practitioners to identify, code and interpret the issues raised within a letter of complaint. Trends on broader constructs can be then developed (eg, problems in bureaucracy and safety incidents), and detailed at a more specific level (ie, subcategory). Furthermore, patient complaint data can be interpreted and analysed through concepts and literatures that appear associated with the category and domain levels. For example, theory on communication and dialogue ${ }^{84}$ compassion and caring, ${ }^{85} 86$ and rule violations ${ }^{87-89}$ may facilitate analysis of 'relationship' problems. Alternatively, human factors theory appears essential for understanding issues relating to safety problems. ${ }^{79}{ }^{80}$ It is notable that the separation of the relational and clinical/management issues corresponds to the sociological literature which describes healthcare in terms of a clash between 'system' (clinical and management) factors and 'lifeworld' (relationship) concerns..$^{90} 91$ Examining the tensions between 'relationships' and 'system' issues within healthcare organisations may be useful for understanding and learning from patient complaints (eg, on how 'systems' shape patient perspectives of care). ${ }^{91} 92$

The taxonomy draws on previous research, for example that which attempted to develop parsimonious codes for analysing patient complaints, ${ }^{63}$ and the conceptual distinctions made between the types of issues raised within a patient complaint. ${ }^{10}{ }^{78} \mathrm{It}$ combines these different approaches, and uses the coded content of 88069 complaints (and the 113551 issues within them) to ensure comprehensiveness. Inconsistencies found across the set of studies, for example, the coding of communication at category and subcategory levels and the use of 'umbrella' concepts, are reduced.

The taxonomy may be beneficial for developing international standards to analyse patient complaints, and would emulate work conducted on adverse event analysis standardisation. ${ }^{93-95}$ For example, systematic frameworks have been developed to standardise the identification and analysis of contributory factors leading to adverse events. ${ }^{96}$ Contributory factors underlying adverse events show them having a high commonality internationally, ${ }^{97}$ and developing a similarly universal taxonomy and procedure for analysing patient complaints would allow for international learning and benchmarking (eg, at present consensus is often lacking between studies on the concepts used to analyse complaints). As indicated by the patient safety literature, ${ }^{3}$ this is likely to focus on different issues than those capture by adverse event analysis taxonomies; for example, subjective concepts such as compassion and sensitivity, which patients view as important, but cannot be easily managed by the organisation $^{98}$ and are not typically investigated through adverse event taxonomies. ${ }^{99}$

\section{Future investigations of patient complaints}

In developing and implementing the coding taxonomy described in figure 4, three stages may be undertaken.

First, the reliability and usability of the complaint coding taxonomy should be assessed; for example, through having different raters use the taxonomy to code a sample of patient complaints. This would allow an assessment of the extent to which information on each domain, category and subcategory can be reliably coded with a letter of complaint.

Second, the taxonomy should be used to structure the analysis and interpretation of patient complaint data. For example, in understanding where within the care process problems occurred (ie, identified by the use of the taxonomy to analyse patient complaints), their severity (eg, threat to patient safety) and their impact upon patient outcomes (eg, harm). An underlying flaw in patient complaint research is the mixing of data on stages of care (eg, examinations, treatment) and more generic problems (eg, communication, staff skills), and further conceptual development is required to better understand how these interact within the taxonomy.

Third, using the taxonomy, patient complaint data might be subject to more sophisticated analyses at the aggregate level; for example, analysing a national 
sample of complaints (to collect norm data, make associations between complaints and care quality at healthcare institutions, compare against existing frameworks). This would allow for healthcare organisations to be compared and for deviations (in comparison with the norm) in particular types of complaint to be identified. The build-up of lower level complaint issues (eg, staff attitudes) within a unit or hospital might be better captured, and used as a potential (or 'early-warning') indicator of poor quality care, as shown with near miss data in the medical error literature. ${ }^{100}$

\section{Study limitations}

The review has a number of limitations. Primarily, our analysis of the issues underlying complaints involved secondary interpretation of the data reviewed in this paper. This was necessary for performing an analysis of trends within the literature, yet involved recoding concepts that in some cases had minimal qualification or definition.

Our analysis of the issues underlying patient complaints is also skewed towards larger studies, and those with multiple patient complaint issue codes are more fully represented. This reflects the differences in size of studies and the depth of their analyses. The complaint coding taxonomy only represents the domains and categories of complaints coded within the literature, and there may be unidentified ones. Additionally, as described above, some categories and subcategories overlap (eg, poor attitudes may be linked to poor communication), and subcategories cannot be completely exclusive.

The literature review largely focuses on studies which presented quantitative data, and future work may wish to incorporate the qualitative studies. ${ }^{19} 20$ In particular, development of the concepts underlying the relationships categories is required, and the use of the safety and quality literature to expand the 'clinical' domain may be beneficial. Furthermore, in some cases, data in patient complaint studies were not fully reported or described clearly, leading to data being not retrievable (table 1). Finally, for the study selection, only the later stages ( 3 and 4) were performed by two raters, with the potential for error or bias being heightened in stages 1 and 2 .

\section{Conclusions}

A range of clinical, management and relationship issues underlie patient complaints. The systematic collation of data on patient complaints potentially provides a mechanism through which the standard of healthcare can be monitored and system-level interventions developed. Although patient complaints provide a unique and unvarnished insight into the problems that occur during healthcare episodes, challenges remain in using the data held within them. In comparison with other forms of quality and safety data (eg, accident and incident data), the methodologies used to analyse patient complaints are inconsistent or do not provide an optimal level of depth into complaints. Furthermore, there is considerable variation in the frameworks used to guide the coding of issues underlying patient complaints. This means that data are unstandardised, difficult to make comparisons of and problematic to demonstrate relationships with. Improvements in the methodology used to codify complaints will help to overcome these issues.

Contributors TWR led the writing of the paper and the analysis of complaint issues. AG led the synthesis of the 59 studies, the amalgamation of their results and contributed to the writing of the paper. JR ran the literature searchers, extracted the data and participated in the writing.

Funding A small grant from the London School of Economics. Competing interests None.

Provenance and peer review Not commissioned; externally peer reviewed.

Data sharing statement Data from the literature review will be available online.

Open Access This is an Open Access article distributed in accordance with the Creative Commons Attribution Non Commercial (CC BY-NC 3.0) license, which permits others to distribute, remix, adapt, build upon this work non-commercially, and license their derivative works on different terms, provided the original work is properly cited and the use is non-commercial. See: http://creativecommons.org/licenses/by-nc/3.0/

\section{REFERENCES}

1 Donaldson L. An organisation with a memory: Learning from adverse events in the NHS. London: Department of Health, 2000.

2 Weingart SN, Pagovich O, Sands DZ, et al. Patient-reported service quality on a medicine unit. Int J Qual Health Care 2006;18:95-101.

3 Levtzion-Korach O, Frankel A, Alcalai H, et al. Integrating incident data from five reporting systems to assess patient safety: making sense of the elephant. Jt Comm J Qual Patient Saf 2010;36:402-10.

4 Weingart SN, Pagovich O, Sands DZ, et al. What can hospitalized patients tell us about adverse events? Learning from patient-reported incidents. J Gen Intern Med 2005;20:830-6.

5 Francis R. Report of the mid-Staffordshire NHS Foundation Trust Public Inquiry. London: The Stationary Office, 2013.

6 Clwyd A, Hart T. A review of the NHS hospitals complaints system: putting patients back in the picture. London, England: Department of Health, 2013.

7 Bark P, Vincent C, Jones A, et al. Clinical complaints: a means of improving quality of care. Qual Health Care 1994;3:123-32.

8 Hsieh SY. An exploratory study of complaints handling and nature. Int J Nurs Pract 2012;18:471-80.

9 Bismark M, Dauer E, Paterson R, et al. Accountability sought by patients following adverse events from medical care: the New Zealand experience. CMAJ 2006;175:889-94.

10 Lloyd-Bostock S, Mulcahy L. The Social Psychology of making and responding to hospital complaints: an account model of complaint processes. Law Policy 1994;16:123-47.

11 Mulcahy L, Tritter J. Pathways, pyramids and icebergs? Mapping the links between dissatisfaction and complaints. Sociol Health Illn 1998;20:825-47. 
12 Carmel S. Patient complaint strategies in a general hospital. Hosp Health Serv Adm 1990;35:277-88.

13 Jangland E, Gunningberg L, Carlsson M. Patients' and relatives' complaints about encounters and communication in health care: evidence for quality improvement. Patient Educ Couns 2009;75:199-204.

14 Reader T, Gillespie A. Patient neglect in healthcare institutions: a systematic review and conceptual model. BMC Health Serv Res 2013;13:156.

15 Taylor-Adams S, Vincent C, Stanhope N. Applying human factors methods to the investigation and analysis of clinical adverse events. Saf Sci 1999;31:143-59.

16 Gawande AA, Studdert D, Zinner M, et al. Analysis of errors reported by surgeons at three teaching hospitals. Surgery 2003;133:614-21.

17 Reader T, Flin R, Lauche K, et al. Non-technical skills in the Intensive Care Unit. Br J Anaesth 2006;96:551-9.

18 Moher D, Liberati A, Tetzlaff J, et al. Preferred reporting items for systematic reviews and meta-analyses: the PRISMA statement. PLoS Med 2009;6:e1000097.

19 Alemi F, Hurd P. Rethinking satisfaction surveys: time to next complaint. Jt Comm J Qual Improv 2009;35:156-61.

20 Mailis-Gagnon A, Nicholson K, Chaparro L. Analysis of complaints to a tertiary care pain clinic over a nine-year period. Pain Res Manag 2010;15:17.

21 Allsop J, Mulcahy L. Dealing with clinical complaints. Qual Health Care 1995;4:135-43.

22 Bismark MM, Spittal MJ, Gogos AJ, et al. Remedies sought and obtained in healthcare complaints. BMJ Qual Saf 2011;20:806-10.

23 Schwartz LR, Overton DT. Emergency department complaints: a one-year analysis. Ann Emerg Med 1987;16:857-61.

24 Chande VT, Bhende MS, Davis HW. Pediatric emergency department complaints: a three-year analysis of sources and trends. Ann Emerg Med 1991;20:1014-16.

25 Owen C. Formal complaints against general practitioners: a study of 1000 cases. Br J Gen Pract 1991;41:113.

26 Burstein J, Fleisher GR. Complaints and compliments in the pediatric emergency department. Pediatr Emerg Care 1991;7:138-40.

27 Hunt M, Glucksman M. A review of 7 years of complaints in an inner-city accident and emergency department. Arch Emerg Med 1991;8:17-23.

28 Donaldson LJ, Cavanagh J. Clinical complaints and their handling: a time for change? Qual Saf Health Care 1992;1:21-5.

29 Kadzombe E, Coals J. Complaints against doctors in an accident and emergency department: a 10-year analysis. Arch Emerg Med 1992;9:134-42.

30 Hanson R, Clifton-Smith B, Fasher B. Patient dissatisfaction in a paediatric accident and emergency department. J Qual Clin Pract 1994;14:137-43.

31 Allsop J. Two sides to every story: complainants' and doctors' perspectives in disputes about medical care in a general practice setting. Law Policy 1994;16:149-83.

32 Nettleton S, Harding G. Protesting patients: a study of complaints submitted to a family health service authority. Sociol Health Illn 1994;16:38-61.

33 Curka PA, Pepe PE, Zachariah BS, et al. Incidence, source, and nature of complaints received in a large, urban emergency medical services system. Acad Emerg Med 1995;2:508-12.

34 Webb B. A study of complaints by patients of different age groups in an NHS trust. Nurs Stand 1995;9:34.
35 Ingram K, Roy L. Complaints against psychiatrists: a five year study. Psychiatric Bulletin 1995;19:620-2.

36 Mulholland J, Dawson K. A complaints management system: strengths and weaknesses. N Engl J Med 1998;111:77.

37 Mace S. An analysis of patient complaints in an observation unit. J Qual Clin Pract 1998;18:151.

38 Lim H, Tan C, Goh L, et al. Why do patients complain? A primary health care study. Singapore Med J 1998;39:390-5.

39 Daniel A, Burn R, Horarik S. Patients' complaints about medical practice. Med J Aust 1999;170:598-602.

40 Pichert JW, Federspiel C, Hickson G, et al. Identifying medical center units with disproportionate shares of patient complaints. Jt Comm J Qual Improv 1999;25:288.

41 Allen LW, Creer E, Leggitt M. Developing a patient complaint tracking system to improve performance. Jt Comm J Qual Improv 2000;26:217-26.

42 Halperin EC. Grievances against physicians: 11 years' experience of a medical society grievance committee. West J Med 2000;173:235.

43 Anderson K, Allan D, Finucane P. Complaints concerning the hospital care of elderly patients: a 12-month study of one hospital's experience. Age Ageing 2000;29:409-12.

44 Pitarka-Carcani I, Szmukler G, Henderson C. Complaints about care in a mental health trust. Psychiatric Bulletin 2000;24:372-6.

45 Anderson K, Allan D, Finucane P. A 30 month study of patient complaints at a major Australian hospital. J Qual Clin Pract 2001;21:109-11.

46 Powers TL, Bendall-Lyon D. Using complaint behavior to improve quality through the structure and process of service delivery. J Consum Satisfaction Dissatisfaction Complaining Behav 2002;15:13-21.

47 Taylor DM, Wolfe R, Cameron PA. Complaints from emergency department patients largely result from treatment and communication problems. Emerg Med 2002;14:43-9.

48 Colwell CB, Pons PT, Pi R. Complaints against an EMS system. J Emerg Med 2003;25:403-8.

49 Choy K, Lau A, Li E, et al. The evolution of complaint management in the Hong Kong Hospital Authority. Part 1: complaints management-a tool for system change? Hong Kong Med J 2004;10:290.

50 Taylor DM, Wolfe RS, Cameron PA. Analysis of complaints lodged by patients attending Victorian hospitals, 1997-2001. Med J Aust 2004;181:31-5.

51 Wofford MM, Wofford JL, Bothra J, et al. Patient complaints about physician behaviors: a qualitative study. Acad Med 2004;79:134-8.

52 Stelfox HT, Gandhi TK, Orav EJ, et al. The relation of patient satisfaction with complaints against physicians and malpractice lawsuits. Am J Med 2005;118:1126-33.

53 Friele RD, Sluijs EM. Patient expectations of fair complaint handling in hospitals: empirical data. BMC Health Serv Res 2006;6:106.

54 Chavan R, Porter C, Sandramouli S. Formal complaints at an eye hospital: a three-year analysis. Clinical Governance: An International Journal 2007;12:85-92.

55 Cowan J, Wilson J. Complaints in primary care arising from bereavement. Clinical Governance: An International Journal 2007;12:296-304.

56 Kline T, Willness C, Ghali W. Predicting patient complaints in hospital settings. Qual Saf Health Care 2008;17:346-50.

57 Saravanan B, Ranganathan E, Jenkinson L. Lessons learnt from complaints by surgical patients. Clinical Governance: An International Journal 2007;12:155-58. 
58 Siyambalapitiya S, Caunt J, Harrison N, et al. A 22 month study of patient complaints at a National Health Service hospital. Int J Nurs Pract 2007;13:107-10.

59 Natangelo R. Clinicians' and managers' responses to patients' complaints: a survey in hospitals of the Milan area. Clinical Governance: An International Journal 2007;12:260-6.

60 Wong L, Ooi S, Goh L. Patients' complaints in a hospital emergency department in Singapore. Singapore Med J 2007;48:990-5.

61 Haw C, Collyer J, Sugarman P. Patients' complaints at a large psychiatric hospital: can they lead to better patient services? Int J Health Care Qual Assur 2010;23:400-9.

62 Friele RD, Sluijs EM, Legemaate J. Complaints handling in hospitals: an empirical study of discrepancies between patients' expectations and their experiences. BMC Health Serv Res 2008;8:199.

63 Montini T, Noble AA, Stelfox HT. Content analysis of patient complaints. Int J Qual Health Care 2008;20:412-20.

64 Giugliani C, Gault N, Fares V, et al. Evolution of patients' complaints in a French university hospital: is there a contribution of a law regarding patients' rights? BMC Health Serv Res 2009;9:141.

65 Parry J, Hewage U. Investigating complaints to improve practice and develop policy. Int J Health Care Qual Assur 2009;22:663-9.

66 Moghadam JM, Ibrahimipour H, Akbari AS, et al. Study of patient complaints reported over 30 months at a large heart centre in Tehran. Qual Saf Health Care 2010;19:1-5.

67 Stimson C, Pichert JW, Moore IN, et al. Medical malpractice claims risk in urology: an empirical analysis of patient complaint data. J Urol 2010;183:1971-6.

68 Hsieh SY. The use of patient complaints to drive quality improvement: an exploratory study in Taiwan. Health Serv Manage Res 2010;23:5-11.

69 McGregor MJ, Cohen M, Stocks-Rankin C-R, et al. Complaints in for-profit, non-profit and public nursing homes in two Canadian provinces. Open Med 2011;5:e183.

70 Gogos AJ, Clark RB, Bismark MM, et al. When informed consent goes poorly: a descriptive study of medical negligence claims and patient complaints. Med J Aust 2011;195:340-4.

71 Källberg A-S, Göransson KE, Östergren J, et al. Medical errors and complaints in emergency department care in Sweden as reported by care providers, healthcare staff, and patients-a national review. Eur J Emerg Med 2013;20:33-8.

72 Sachdeo A, Konfino S, Icyda RU, et al. An analysis of patient grievances in a dental school clinical environment. J Dent Educ 2012;76:1317-22.

73 Schnitzer S, Kuhlmey A, Adolph H, et al. Complaints as indicators of health care shortcomings: which groups of patients are affected? Int J Qual Health Care 2012;24:476-82.

74 Mann CD, Howes JA, Buchanan A, et al. One-year audit of complaints made against a University Hospital Surgical Department. ANZ J Surg 2012;82:671-4.

75 van Mook WN, Gorter SL, Kieboom W, et al. Poor professionalism identified through investigation of unsolicited healthcare complaints. Postgrad Med J 2012;88:443-50.

76 Bismark MM, Spittal MJ, Gurrin LC, et al. Identification of doctors at risk of recurrent complaints: a national study of healthcare complaints in Australia. BMJ Qual Saf 2013;22:532-40.

77 Doron I, Gal I, Shavit M, et al. Unheard voices: complaint patterns of older persons in the health care system. Eur J Ageing 2011;8:63-71.
78 Powers T, Bendall-Lyons D. Using complaint behaviour to improve quality through the structure and process of service delivery. J Consum Satisf Dissatisf Complain Behav 2002;15:14-21.

79 Pronovost P, Wachter R. Balancing "no blame" with accountability in patient safety. N Engl J Med 2010;361:1401-6.

80 Vincent C. Patient safety. London: Elsevier, 2006.

81 Ferlie E, Shortell S. Improving the quality of health care in the United Kingdom and the United States: a framework for change. Milbank Q 2001;79:281-315.

82 Swayne LE, Duncan WJ, Ginter PM. Strategic management of health care organizations. John Wiley \& Sons, 2012.

83 Kenagy J, Berwick D, Shore M. Service quality in health care. JAMA 1999;281:661-5.

84 Gillespie A, Cornish F. Intersubjectivity: towards a dialogical analysis. J Theory Soc Behav 2010;40:19-46.

85 Bradshaw A. Measuring nursing care and compassion: the McDonaldised nurse? J Med Ethics 2009;35:465-8.

86 Maslach C, Jackson S. Burnout in organizational settings. Appl Soc Psychol 1984;5:133-53.

87 Jewkes R, Abrahams N, Mvo Z. Why do nurses abuse patients? Reflections from South African obstetric services. Soc Sci Med 1998;47:1781-95.

88 Robinson S, Bennett R. A typology of deviant workplace behaviors: A multidimensional scaling study. Acad Manage J 1995;38:555-72.

89 Lawton R. Not working to rule: understanding procedural violations at work. Saf Sci 1998;28:77-95.

90 de Vries E, Ramrattan M, Smorenburg S, et al. The incidence and nature of in-hospital adverse events: a systematic review. Qual Saf Health Care 2008;17:216-23.

91 Greenhalgh T, Robb N, Scambler G. Communicative and strategic action in interpreted consultations in primary health care: A Habermasian perspective. Soc Sci Med 2006;63:1170-87.

92 Habermas J. The theory of communicative action: Volume 2: lifeword and system: a critique of functionalist reason. Beacon press, 1985.

93 Runciman W, Hibbert P, Thomson R, et al. Towards an international classification for patient safety: key concepts and terms. Int J Qual Health Care 2009;21:18-26.

94 Runciman WB, Baker GR, Michel P, et al. Tracing the foundations of a conceptual framework for a patient safety ontology. Qual Saf Health Care 2010;19:1-5.

95 Sherman H, Castro G, Fletcher M, et al. Towards an international classification for patient safety: the conceptual framework. Int J Qual Health Care 2009;21:2-8.

96 Lawton R, McEachan RR, Giles SJ, et al. Development of an evidence-based framework of factors contributing to patient safety incidents in hospital settings: a systematic review. BMJ Qual Saf 2012;21:369-80.

97 Pham JC, Aswani MS, Rosen M, et al. Reducing medical errors and adverse events. Annu Rev Med 2012;63:447-63.

98 Reader T, Gillespie A, Mannell J. Patient neglect in 21st century healthcare institutions: a community health psychology perspective. J Health Psychol 2014;19:137-48.

99 Chang A, Schyve PM, Croteau RJ, et al. The JCAHO patient safety event taxonomy: a standardized terminology and classification schema for near misses and adverse events. Int J Qual Health Care 2005;17:95-105.

100 Catchpole K, Giddings A, Wilkinson M, et al. Improving patient safety by identifying latent failures in successful operations. Surgery 2007;142:102-10. 